\title{
论 文
}

\section{倒向随机 Volterra 积分方程适应解的表示}

献给李大潜教授 80 华诞

\section{㢕烔敏 1,2}

1. 复旦大学数学科学学院, 上海 200433 ;

2. Department of Mathematics, University of Central Florida, Orlando, FL 32816, USA

E-mail: jiongmin.yong@ucf.edu

收稿日期: 2016-02-16; 接受日期: 2016-07-29; 网络出版日期: 2016-12-12

美国国家科学基金 (批准号: DMS-1406776) 资助项目

摘要 倒向随机 Volterra 积分方程可以看作 (确定性) Volterra 积分方程和倒向随机微分方程的推广, 在随机最优控制理论和数学金融学中有诸多应用. 本文利用正倒向随机微分方程适应解表示的思想, 得到所研究的一类倒向随机 Volterra 积分方程适应解的表示. 这样的结果对研究适应解的正则性以 及数值计算有重要的意义.

关键词 倒向随机 Volterra 积分方程 适应解 正倒向随机微分方程

MSC (2010) 主题分类 $60 \mathrm{H} 20,60 \mathrm{H} 10,45 \mathrm{D} 05$

\section{1 引言}

考虑一个带流的完备概率空间 $(\Omega, \mathcal{F}, \mathbb{F}, \mathbb{P})$ 和在此空间上定义的一个标准的一维 Brown 运动 $W(\cdot)$. 假定 $\mathbb{F}=\left\{\mathcal{F}_{t}\right\}_{t \geqslant 0}$ 就是 $W(\cdot)$ 的自然 $\sigma$ - 域流的完备化. 考虑下述积分方程:

$$
Y(t)=\psi(t)+\int_{t}^{T} g(t, s, Y(s), Z(t, s), Z(s, t)) d s-\int_{t}^{T} Z(t, s) d W(s), \quad t \in[0, T] .
$$

我们称如上的方程为倒向随机 Volterra 积分方程 (简称 BSVIE), 其中 $g$ 是一个给定的随机函数, 称为 该方程的系数 (也称生成子 (generator)), $\psi$ 是一个 $\mathcal{F}_{T^{-}}$可测的随机过程 (未必 $\mathbb{F}$ - 适应), 称为自由项. 与 (1.1) 有密切关系的是如下方程:

$$
Y(t)=\xi+\int_{t}^{T} g(s, Y(s), Z(s)) d s-\int_{t}^{T} Z(s) d W(s), \quad t \in[0, T] .
$$

这样的方程称为倒向随机微分方程 (简称 BSDE). 其实, (1.2) 是 BSDE 的积分形式. 易见, (1.1) 是 (1.2) 的一个自然推广. 线性 BSDE 是 Bismut ${ }^{[1]}$ 在 1973 年研究随机线性二次最优控制时首次引入的. 1990 年, Pardoux 和 Peng ${ }^{[2]}$ 开始了一般 (非线性) BSDE 的研究. 部分后续的工作可以参见文献 [3-9]. 
2002 年, 作为 BSDE 的推广, Lin ${ }^{[10]}$ 首次研究了比 (1.1) 更为特殊的一类 BSVIE. 以 (正向) 随机 Volterra 积分方程最优控制的最大值原理和资产组合过程动态风险作为背景, Yong ${ }^{[11-13]}$ 首次研究了 形如 (1.1) 的 BSVIE. 根据文献 [13], 在适当的条件下, 方程 (1.1) 存在唯一的 $M$ - 适应解 $(Y(\cdot), Z(\cdot, \cdot))$, 即对任何的 $t \in[0, T)$, 映照 $s \mapsto(Y(s), Z(t, s))$ 在 $[t, T]$ 上是 $\mathbb{F}$ - 适应的, 方程 (1.1) 在普通的 Itô 意义 下得以满足, 且有下述鞅表示等式:

$$
Y(t)=\mathbb{E}[Y(t)]+\int_{0}^{t} Z(t, s) d W(s), \quad t \in[0, T] .
$$

有关 BSVIE 的某些正则性的研究和比较定理的建立可参见文献 $[13,14]$. 其他相关的研究可以参见文 献 $[15,16]$.

我们注意到, 在文献 [13] 中, 为了获得若干正则性的结果, 对系数 $g$ 和自由项 $\psi$ 施加了一些涉及 Malliavin 可导性的条件. 在实际应用中, 人们可能不希望有那样的条件限制. 同时, 在文献 [13] 中出现 的需要 Malliavin 可导性条件才能保证的正则性结果在数学上的表述也欠漂亮. 受有关正倒向随机微 分方程 (简称 FBSDE) 适应解表示 (参见文献 $[4,5]$ ) 的启示, 我们期望对某些 BSVIE, 得到其适应解的 某种表示, 以方便得到相关的正则性, 并对 BSVIE 的解的数值计算提供方便. 本文是第一次对 BSVIE 作这样的尝试, 故我们的结果还不十分理想.

我们引入下述正向随机微分方程 (简称 FSDE):

$$
\left\{\begin{array}{l}
d X(t)=b(t, X(t)) d t+\sigma(t, X(t)) d W(t), \quad t \in[0, T], \\
X(0)=x,
\end{array}\right.
$$

其中 $b, \sigma:[0, T] \times \mathbb{R} \rightarrow \mathbb{R}$ 是两个给定的函数, 而 $x \in \mathbb{R}$. 我们考虑如下一类特殊的 BSVIE:

$$
Y(t)=\psi(t, X(T))+\int_{t}^{T} g(t, s, X(s), Y(s), Z(t, s)) d s-\int_{t}^{T} Z(t, s) d W(s), \quad t \in[0, T],
$$

其中 $g: \Delta \times \mathbb{R}^{3} \rightarrow \mathbb{R}, \psi:[0, T] \times \mathbb{R} \rightarrow \mathbb{R}$ 为两个确定性函数, 而

$$
\Delta=\{(t, s) \mid 0 \leqslant t \leqslant s \leqslant T\} .
$$

易见, BSVIE (1.4) 中系数和自由项的随机性全部来自于 FSDE (1.3) 的解 $X(\cdot)$. 在应用中, 人们通常 用形如 (1.3) 的 (正向) 随机微分方程来描述经济因子 (economic factors)、资产价格过程和财富过程 等. 而 BSVIE (1.4) 可以用来描述动态风险和动态/递归效用等. 因此, 如上形式的 BSVIE 中出现的 随机性在许多应用中已经足够了. 本文的主要结果是下面的表示定理.

定理 1.1 在适当的条件下, BSVIE (1.4) 的适应解 $(Y(\cdot), Z(\cdot, \cdot))$ 具有如下的表现形式:

$$
\left\{\begin{array}{l}
Y(s)=\Theta(s, s, X(s)), \\
Z(t, s)=\sigma(s, X(s)) \Theta_{x}(t, s, X(s)),
\end{array}(t, s) \in \Delta,\right.
$$

其中 $X(\cdot)$ 是 $(1.3)$ 的解, $(s, x) \mapsto \Theta(t, s, x)$ 满足如下的带参数 $t$ 的偏微分方程:

$$
\left\{\begin{array}{l}
\Theta_{s}(t, s, x)+\frac{1}{2} \sigma(s, x)^{2} \Theta_{x x}(t, s, x)+b(s, x) \Theta_{x}(t, s, x) \\
\quad+g\left(t, s, x, \Theta(s, s, x), \Theta_{x}(t, s, x) \sigma(s, x)\right)=0, \quad(t, s, x) \in \Delta \times \mathbb{R}, \\
\Theta(t, T, x)=\psi(t, x), \quad(t, x) \in[0, T] \times \mathbb{R} .
\end{array}\right.
$$


我们知道, 直接从 BSVIE 出发很难得到其适应解的正则性, 尤其是映照 $s \mapsto Z(t, s)$ 的正则性. 而 从表示式 (1.5), 人们可以清楚地看到 $(Y(\cdot), Z(\cdot, \cdot))$ 的正则性. 形如 (1.5) 的表示式为 BSVIE 的数值计 算也提供了便利. 可以预见, 我们的结果对有关时间不一致随机最优控制、动态风险度量和 BSVIE 的 数值计算等方面的研究将具有重要意义. 我们会在将来的工作中加以展开.

为了表述方便起见 (及限于篇幅), 本文主要讨论空间维数为 1 、Brown 运动的维数也为 1 的情形. 高维空间和多维 Brown 运动的情形将只作简单叙述. 我们将着重阐述思想方法, 将略去有些冗长的证 明. 详尽的和更一般的讨论, 我们将在另文中给出. 我们还应注意到, 与 BSVIE (1.1) 相比, BSVIE (1.4) 中不含 $Z(s, t)$ 项. 因此, 一个自然的问题是, 假如 $Z(s, t)$ 也出现在 (1.4) 中, 我们是否有同样或类似的 结果? 关于此点, 我们期望在将来的研究中再行讨论.

本文余下部分是这样安排的: 第 2 节将讨论 BSVIE 的逼近; 第 3 节将导出 BSVIE 适应解的表 示; 最后将简略讨论若干可能的推广情形.

\section{2 倒向随机 Volterra 积分方程的逼近}

本节将建立 BSVIE 的逼近定理. 首先, 让我们先引入有关的假设条件.

(H1) 函数 $b, \sigma:[0, T] \times \mathbb{R} \rightarrow \mathbb{R}$ 是连续的, 且存在常数 $L, \sigma_{0}>0$ 使得

$$
\begin{aligned}
& \left|b\left(t, x_{1}\right)-b\left(t, x_{2}\right)\right|+\left|\sigma\left(t, x_{1}\right)-\sigma\left(t, x_{2}\right)\right| \leqslant L\left|x_{1}-x_{2}\right|, \quad \forall t \in[0, T], \quad x_{1}, x_{2} \in \mathbb{R}, \\
& \sigma(t, x) \geqslant \sigma_{0}, \quad \forall(t, x) \in[0, T] \times \mathbb{R} .
\end{aligned}
$$

(H2) 函数 $g: \Delta \times \mathbb{R}^{3} \rightarrow \mathbb{R}$ 和 $\psi:[0, T] \times \mathbb{R} \rightarrow \mathbb{R}$ 是连续的, 且存在常数 $L>0$ 使得

$$
\begin{aligned}
& \left|g\left(t_{1}, s, x, y_{1}, z_{1}\right)-g\left(t_{2}, s, x, y_{2}, z_{2}\right)\right| \leqslant L\left(\left|t_{1}-t_{2}\right|+\left|y_{1}-y_{2}\right|+\left|z_{1}-z_{2}\right|\right), \\
& \quad \forall\left(t_{1}, s\right),\left(t_{2}, s\right) \in \Delta, \quad x, y_{1}, y_{2}, z_{1}, z_{2} \in \mathbb{R}, \\
& \left|\psi\left(t_{1}, x\right)-\psi\left(t_{2}, x\right)\right| \leqslant L\left|t_{1}-t_{2}\right|, \quad \forall t_{1}, t_{2} \in[0, T], \quad x \in \mathbb{R} .
\end{aligned}
$$

我们知道, 当 (H1) 成立时, FSDE (1.3) 存在唯一的强解 $X(\cdot)$; 当 (H2) 成立时, BSVIE (1.4) 存在 唯一的适应解 $(Y(\cdot), Z(\cdot, \cdot))$. 值得指出, 由于在 BSVIE (1.4) 的漂移项中, $Z(s, t)$ 不出现, 因此, 所谓的 $M$ - 适应解的概念在这里是没有必要的. 我们只需 $Z(t, s)$ 对于 $0 \leqslant t \leqslant s \leqslant T$ 有定义即可.

记 $\mathcal{P}[0, T]$ 为所有形如下述的 $[0, T]$ 的分割全体:

$$
\Pi: 0=t_{0}<t_{1}<\cdots<t_{N-1}<t_{N}=T .
$$

并记

$$
\|\Pi\|=\max _{0 \leqslant k \leqslant N-1}\left(t_{k+1}-t_{k}\right), \quad \forall \Pi \in \mathcal{P}[0, T],
$$

称之为分割 $\Pi$ 的长度. 最简单的分割是等长度的分割, 即

$$
t_{k}=\frac{k T}{N}, \quad 0 \leqslant k \leqslant N .
$$

此时,

$$
\|\Pi\|=\frac{T}{N}=t_{k+1}-t_{k}, \quad 0 \leqslant k \leqslant N-1 .
$$


一般而言, 我们并不要求这一点. 不过, 我们可以要求存在一个 $\delta>0$ 使得

$$
\min _{0 \leqslant k \leqslant N-1}\left(t_{k+1}-t_{k}\right) \geqslant \delta\|\Pi\| .
$$

现设 $\Pi \in \mathcal{P}[0, T]$ 是一个形如 $(2.1)$ 的 $[0, T]$ 的分割. 在下面的讨论中, 我们假设 (H1) 和 (H2) 成立. 我 们希望构造一个过程 $\left(Y^{\Pi}(\cdot), Z^{\Pi}(\cdot, \cdot)\right)$, 在某种意义下逼近 $(Y(\cdot), Z(\cdot, \cdot))$, 且 $\left(Y^{\Pi}(\cdot), Z^{\Pi}(\cdot)\right)$ 可用倒向随 机微分方程 (简称 BSDE) 来描述.

首先, 在 $\left[t_{N-1}, T\right]$ 上引入 $\mathrm{BSDE}$ :

$$
\left\{\begin{array}{l}
d Y^{N-1}(s)=-g\left(t_{N-1}, s, X(s), Y^{N-1}(s), Z^{N-1}(s)\right) d s+Z^{N-1}(s) d W(s), \quad s \in\left[t_{N-1}, T\right], \\
Y^{N-1}(T)=\psi\left(t_{N-1}, X(T)\right),
\end{array}\right.
$$

在 (H2) 的条件下, 上述方程存在唯一的适应解 $\left(Y^{N-1}(\cdot), Z^{N-1}(\cdot)\right)$. 然后在 $\left[t_{N-2}, T\right]$ 上 (而不是在 $\left[t_{N-2}, t_{N-1}\right]$ 上) 引入一个特别的 BSDE:

$$
\left\{\begin{array}{l}
d Y^{N-2}(s)=-g\left(t_{N-2}, s, X(s), Y^{N-1}(s), Z^{N-2}(s)\right) d s+Z^{N-2}(s) d W(s), \quad s \in\left(t_{N-1}, T\right], \\
d Y^{N-2}(s)=-g\left(t_{N-2}, s, X(s), Y^{N-2}(s), Z^{N-2}(s)\right) d s+Z^{N-2}(s) d W(s), \quad s \in\left[t_{N-2}, t_{N-1}\right], \\
Y^{N-2}(T)=\psi\left(t_{N-2}, X(T)\right), \quad Y^{N-2}\left(t_{N-1}\right)=Y^{N-2}\left(t_{N-1}+0\right) .
\end{array}\right.
$$

注意, 上述方程中, 在 $\left(t_{N-1}, T\right]$ 上, $Y^{N-1}(s)$ 已经由 (2.3) 确定, 不可更改. 但是, 由于 $g$ 中出现的是 $t_{N-2}$, 而不是 $t_{N-1}$, 故在 $\left(t_{N-1}, T\right]$ 上, $\left(Y^{N-2}(\cdot), Z^{N-2}(\cdot)\right)$ 和 $\left(Y^{N-1}(\cdot), Z^{N-1}(\cdot)\right)$ 满足的方程不同. 从 而, 即便 $Y^{N-1}(s)$ 出现在方程的飘移项中, 相应的 $Z^{N-2}(\cdot)$ 多半也是与 $Z^{N-1}(\cdot)$ 不同的. 在我们的假 设条件下, 上述 BSDE 在 $\left[t_{N-2}, T\right]$ 存在唯一的适应解 $\left(Y^{N-2}(\cdot), Z^{N-2}(\cdot)\right)$.

一般地, 我们在 $\left[t_{k}, T\right]$ 上引入下述 BSDE:

$$
\begin{cases}d Y^{k}(s)=-g\left(t_{k}, s, X(s), Y^{\ell}(s), Z^{k}(s)\right) d s+Z^{k}(s) d W(s), & s \in\left(t_{\ell}, t_{\ell+1}\right], \quad k+1 \leqslant \ell \leqslant N-1, \\ d Y^{k}(s)=-g\left(t_{k}, s, X(s), Y^{k}(s), Z^{k}(s)\right) d s+Z^{k}(s) d W(s), & s \in\left[t_{k}, t_{k+1}\right], \\ Y^{k}(T)=\psi\left(t_{k}, X(T)\right), \quad Y^{k}\left(t_{\ell}\right)=Y^{k}\left(t_{\ell}+0\right), & k+1 \leqslant \ell \leqslant N-1 .\end{cases}
$$

类似于上述 $(2.4)$, 对于每个 $\ell \geqslant k+1$, 在 $\left(t_{\ell}, t_{\ell+1}\right]$ 上, 方程漂移项中出现的是 $Y^{\ell}(\cdot)$, 而不是 $Y^{k}(\cdot)$. 在 假设 (H1) 和 (H2) 下, 上述 BSDE 存在唯一的适应解. 我们定义

$$
\begin{cases}Y^{\Pi}(s)=Y^{0}(s) I_{\left[0, t_{1}\right]}(s)+\sum_{k=1}^{N-1} Y^{k}(s) I_{\left(t_{k}, t_{k+1}\right]}(s), & s \in[0, T] \\ Z^{\Pi}(t, s)=Z^{0}(s) I_{\left[0, t_{1}\right]}(t)+\sum_{k=1}^{N-1} Z^{k}(s) I_{\left(t_{k}, t_{k+1}\right]}(t), & 0 \leqslant t \leqslant s \leqslant T\end{cases}
$$

按上述方式定义的 $Y^{\Pi}(\cdot)$ 在 $t_{N-1}, t_{N-2}, \ldots, t_{1}$ 处有可能间断. 令

$$
\tau^{\Pi}(t)=t_{k} I_{\left[t_{k}, t_{k+1}\right]}(t)+\sum_{\ell=k+1}^{N-1} t_{\ell} I_{\left(t_{\ell}, t_{\ell+1}\right]}(t), \quad t \in[0, T]
$$


则有

$$
0 \leqslant t-\tau^{\Pi}(t) \leqslant\|\Pi\|, \quad t \in[0, T]
$$

并且 (2.5) 可写成

$$
\left\{\begin{array}{l}
d Y^{k}(s)=-g\left(t_{k}, s, X(s), Y^{\Pi}(s), Z^{k}(s)\right) d s+Z^{k}(s) d W(s), \quad s \in\left[t_{k}, T\right], \\
Y^{k}(T)=\psi\left(t_{k}, X(T)\right) .
\end{array}\right.
$$

同时, 对于任何 $t \in(0, T]$, 设 $t \in\left(t_{k}, t_{k+1}\right]$, 有下述结果:

$$
\begin{aligned}
Y^{\Pi}(t)= & Y^{k}(t) \\
= & \psi\left(t_{k}, X(T)\right)+\int_{t}^{t_{k+1}} g\left(t_{k}, s, X(s), Y^{k}(s), Z^{k}(s)\right) d s \\
& +\sum_{\ell=k+1}^{N-1} \int_{t_{\ell}}^{t_{\ell+1}} g\left(t_{k}, s, X(s), Y^{\ell}(s), Z^{k}(s)\right) d s-\int_{t}^{T} Z^{k}(s) d W(s) \\
= & \psi\left(\tau^{\Pi}(t), X(T)\right)+\int_{t}^{T} g\left(\tau^{\Pi}(t), s, X(s), Y^{\Pi}(s), Z^{\Pi}(t, s)\right) d s-\int_{t}^{T} Z^{\Pi}(t, s) d W(s) .
\end{aligned}
$$

所以, $\left(Y^{\Pi}(\cdot), Z^{\Pi}(\cdot)\right)$ 在每个 $\left(t_{k}, t_{k+1}\right)$ 区间上满足一个 BSDE, 而在 $[0, T]$ 上又满足一个 BSVIE. 对于 上面构造的 $\left(Y^{\Pi}(\cdot), Z^{\Pi}(\cdot, \cdot)\right)$, 我们有下述定理.

定理 2.1 假定 $(\mathrm{H} 1)$ 和 $(\mathrm{H} 2)$ 成立, $\left(Y^{\Pi}(\cdot), Z^{\Pi}(\cdot, \cdot)\right)$ 由上构造, 则

$$
\mathbb{E} \int_{0}^{T}\left|Y^{\Pi}(t)-Y(t)\right|^{2} d t+\mathbb{E} \int_{0}^{T} \int_{t}^{T}\left|Z^{\Pi}(t, s)-Z(t, s)\right|^{2} d s d t \leqslant K\|\Pi\|^{2} .
$$

证明 利用文献 [13] 中的结果, 综合 (H2), 我们有

$$
\begin{aligned}
& \mathbb{E} \int_{0}^{T}\left|Y^{\Pi}(t)-Y(t)\right|^{2} d t+\mathbb{E} \int_{0}^{T} \int_{t}^{T}\left|Z^{\Pi}(t, s)-Z(t, s)\right|^{2} d s d t \\
& \leqslant K \mathbb{E}\left(\left|\psi\left(\tau^{\Pi}(t), X(T)\right)-\psi(t, X(T))\right|^{2}+\int_{0}^{T} \int_{t}^{T} \mid g\left(\tau^{\Pi}(t), s, X(s), Y(s), Z(t, s)\right)\right. \\
& \left.\quad-\left.g(t, s, X(s), Y(s), Z(t, s))\right|^{2} d s d t\right) \\
& \quad \leqslant K\|\Pi\|^{2}
\end{aligned}
$$

这就给出了 BSVIE 的适应解被一族 BSDE 的适应解逼近.

\section{3 适应解的表示}

本节将导出 BSVIE (1.4) 适应解的表示. 为此, 我们先在 $\left[t_{N-1}, T\right]$ 上考虑下述解耦的 FBSDE:

$$
\left\{\begin{array}{l}
d X(s)=b(s, X(s)) d s+\sigma(s, X(s)) d W(s) \\
d \bar{Y}^{N-1}(s)=-g\left(t_{N-1}, s, X(s), \bar{Y}^{N-1}(s), \bar{Z}^{N-1}(s)\right) d s+\bar{Z}^{N-1}(s) d W(s), \\
\bar{Y}^{N-1}(T)=\psi\left(t_{N-1}, X(T)\right) .
\end{array}\right.
$$


比较 (2.3) 可知,

$$
\left(\bar{Y}^{N-1}(s), \bar{Z}^{N-1}(s)\right)=\left(Y^{N-1}(s), Z^{N-1}(s)\right), \quad s \in\left(t_{N-1}, T\right] .
$$

由文献 $[4,5]$ 知, 在 $(\mathrm{H} 1)$ 和 $(\mathrm{H} 2)$ 条件假设下, 下述表示成立:

$$
\left\{\begin{array}{l}
Y^{N-1}(s)=\bar{Y}^{N-1}(s)=\Theta^{N-1}(s, X(s)), \\
Z^{N-1}(s)=\bar{Z}^{N-1}(s)=\sigma(s, X(s)) \Theta_{x}^{N-1}(s, X(s)),
\end{array} s \in\left[t_{N-1}, T\right],\right.
$$

其中 $\Theta^{N-1}(\cdot, \cdot)$ 是下述抛物型偏微分方程的解:

$$
\begin{cases}\Theta_{s}^{N-1}(s, x)+\frac{1}{2} \sigma(s, x)^{2} \Theta_{x x}^{N-1}(s, x)+b(s, x) \Theta_{x}^{N-1}(s, x) & \\ \quad+g\left(t_{N-1}, s, x, \Theta^{N-1}(s, x), \sigma(s, x) \Theta_{x}^{N-1}(s, x)\right)=0, & (s, x) \in\left[t_{N-1}, T\right] \times \mathbb{R}, \\ \Theta^{N-1}(T, x)=\psi\left(t_{N-1}, x\right), & x \in \mathbb{R} .\end{cases}
$$

接着, 在 $\left[t_{N-2}, T\right]$ 上, 我们考虑 $\mathrm{FBSDE}$

$$
\begin{cases}d X(s)=b(s, X(s)) d s+\sigma(s, X(s)) d W(s), & s \in\left[t_{N-2}, T\right], \\ d \bar{Y}^{N-2}(s)=-g\left(t_{N-2}, s, X(s), \Theta^{N-1}(s, X(s)), \bar{Z}^{N-2}(s)\right) d s+\bar{Z}^{N-2}(s) d W(s), & s \in\left(t_{N-1}, T\right], \\ d \bar{Y}^{N-2}(s)=-g\left(t_{N-2}, s, X(s), \bar{Y}^{N-2}(s), \bar{Z}^{N-2}(s)\right) d s+\bar{Z}^{N-2}(s) d W(s), & s \in\left[t_{N-2}, t_{N-1}\right], \\ \bar{Y}^{N-2}(T)=\psi\left(t_{N-2}, X(T)\right), & \bar{Y}^{N-2}\left(t_{N-1}\right)=\Theta^{N-1}\left(t_{N-1}, X\left(t_{N-1}\right)\right) .\end{cases}
$$

此处, 我们用到了 $\bar{Y}^{N-1}(\cdot)$ 在 $\left[t_{N-1}, T\right]$ 上的表示 (见 (3.2)). 于是, 比较 (2.4) 和 (3.4), 我们得到

$$
\left(\bar{Y}^{N-2}(s), \bar{Z}^{N-2}(s)\right)=\left(Y^{N-2}(s), Z^{N-2}(s)\right), \quad s \in\left[t_{N-2}, T\right] .
$$

令

$$
\bar{Y}^{N-2}(s)=\Theta^{N-2}(s, X(s)), \quad s \in\left[t_{N-2}, T\right],
$$

则在 $\left(t_{N-1}, T\right]$ 上, 我们有

$$
\begin{aligned}
& -g\left(t_{N-2}, s, X(s), \Theta^{N-1}(s, X(s)), \bar{Z}^{N-2}(s)\right) d s+\bar{Z}^{N-2}(s) d W(s)=d \bar{Y}^{N-2}(s) \\
& =\left[\Theta_{s}^{N-2}(s, X(s))+\frac{1}{2} \sigma(s, X(s))^{2} \Theta_{x x}^{N-2}(s, X(s))+b(s, X(s)) \Theta_{x}^{N-2}(s, X(s))\right] d s \\
& \quad+\sigma(s, X(s)) \Theta_{x}^{N-2}(s, X(s)) d W(s) .
\end{aligned}
$$

因此,

$$
\bar{Z}^{N-2}(s)=\sigma(s, X(s)) \Theta_{x}^{N-2}(s, X(s)), \quad s \in\left(t_{N-1}, T\right]
$$

同时，

$$
\begin{aligned}
& \Theta_{s}^{N-2}(s, X(s))+\frac{1}{2} \sigma(s, X(s))^{2} \Theta_{x x}^{N-2}(s, X(s))+b(s, X(s)) \Theta_{x}^{N-2}(s, X(s)) \\
& \quad+g\left(t_{N-2}, s, X(s), \Theta^{N-1}(s, X(s)), \sigma(s, X(s)) \Theta_{x}^{N-2}(s, X(s))=0, \quad s \in\left(t_{N-1}, T\right],\right.
\end{aligned}
$$


并且, 我们施加终值条件

$$
\Theta^{N-2}(T, X(T))=\psi\left(t_{N-2}, X(T)\right) .
$$

而在 $\left[t_{N-2}, t_{N-1}\right]$ 上, 我们有

$$
\begin{aligned}
- & g\left(t_{N-2}, s, X(s), \bar{Y}^{N-2}(s), \bar{Z}^{N-2}(s)\right) d s+\bar{Z}^{N-2}(s) d W(s) \\
= & d \bar{Y}^{N-2}(s) \\
= & {\left[\Theta_{s}^{N-2}(s, X(s))+\frac{1}{2} \sigma(s, X(s))^{2} \Theta_{x x}^{N-2}(s, X(s))+b(s, X(s)) \Theta_{x}^{N-2}(s, X(s))\right] d s } \\
& +\sigma(s, X(s)) \Theta_{x}^{N-2}(s, X(s)) d W(s) .
\end{aligned}
$$

因此,

$$
\bar{Z}^{N-2}(s)=\sigma(s, X(s)) \Theta_{x}^{N-2}(s, X(s)), \quad s \in\left[t_{N-2}, t_{N-1}\right],
$$

同时，

$$
\begin{aligned}
& \Theta_{s}^{N-2}(s, X(s))+\frac{1}{2} \sigma(s, X(s))^{2} \Theta_{x x}^{N-2}(s, X(s))+b(s, X(s)) \Theta_{x}^{N-2}(s, X(s)) \\
& \quad+g\left(t_{N-2}, s, X(s), \Theta^{N-2}(s, X(s)), \sigma(s, X(s)) \Theta_{x}^{N-2}(s, X(s))=0\right.
\end{aligned}
$$

并且, 在 $t_{N-1}$ 处施加自然的终值条件

$$
\Theta^{N-2}\left(t_{N-1}, X\left(t_{N-1}\right)\right)=\Theta^{N-2}\left(t_{N-1}+0, X\left(t_{N-1}\right)\right) .
$$

因此, 我们得到

$$
\left\{\begin{array}{l}
\Theta_{s}^{N-2}(s, x)+\frac{1}{2} \sigma(s, x)^{2} \Theta_{x x}^{N-2}(s, x)+b(s, x) \Theta_{x}^{N-2}(s, x) \\
\quad+g\left(t_{N-2}, s, x, \Theta^{N-1}(s, x), \sigma(s, x) \Theta_{x}^{N-2}(s, x)\right)=0, \quad(s, x) \in\left(t_{N-1}, T\right], \\
\Theta_{s}^{N-2}(s, x)+\frac{1}{2} \sigma(s, x)^{2} \Theta_{x x}^{N-2}(s, x)+b(s, x) \Theta_{x}^{N-2}(s, x) \\
\quad+g\left(t_{N-2}, s, x, \Theta^{N-2}(s, x), \sigma(s, x) \Theta_{x}^{N-2}(s, x)\right)=0, \quad(s, x) \in\left[t_{N-2}, t_{N-1}\right] \times \mathbb{R}, \\
\Theta^{N-2}(T, x)=\psi\left(t_{N-2}, x\right), \quad \Theta^{N-2}\left(t_{N-1}, x\right)=\Theta^{N-2}\left(t_{N-1}+0, x\right), \quad x \in \mathbb{R} .
\end{array}\right.
$$

当上述偏微分方程存在经典解时, $\left(\bar{Y}^{N-1}(\cdot), \bar{Z}^{N-1}(\cdot)\right)$ 具有如下表示:

$$
\left\{\begin{array}{l}
Y^{N-2}(s)=\bar{Y}^{N-2}(s)=\Theta^{N-2}(s, X(s)), \\
Z^{N-2}(s)=\bar{Z}^{N-2}(s)=\sigma(s, X(s)) \Theta_{x}^{N-2}(s, X(s)),
\end{array} \quad s \in\left[t_{N-2}, T\right] .\right.
$$

归纳地, 在 $\left[t_{k}, T\right]$ 上, 我们考虑下述 FBSDE:

$$
\left\{\begin{array}{l}
d X(s)=b(s, X(s)) d s+\sigma(s, X(s)) d W(s), \quad s \in\left[t_{k}, T\right], \\
d \bar{Y}^{k}(s)=-g\left(t_{k}, s, X(s), \Theta^{\ell}(s, X(s)), \bar{Z}^{k}(s)\right) d s+\bar{Z}^{k}(s) d W(s), \\
\quad s \in\left(t_{\ell}, t_{\ell+1}\right], \quad k+1 \leqslant \ell \leqslant N-1, \\
d \bar{Y}^{k}(s)=-g\left(t_{k}, s, X(s), \bar{Y}^{k}(s), \bar{Z}^{k}(s)\right) d s+\bar{Z}^{k}(s) d W(s), \quad s \in\left[t_{k}, t_{k+1}\right], \\
\bar{Y}^{k}(T)=\psi\left(t_{k}, X(T)\right) .
\end{array}\right.
$$


在上面方程中, $\Theta^{\ell}(\cdot, \cdot)$ 在 $\left[t_{\ell}, T\right]$ 上满足一个偏微分方程, 且使得

$$
\left\{\begin{array}{l}
Y^{\ell}(s)=\bar{Y}^{\ell}(s)=\Theta^{\ell}(s, X(s)), \\
Z^{\ell}(s)=\bar{Z}^{\ell}(s)=\sigma(s, X(s)) \Theta^{\ell}(s, X(s)),
\end{array} \quad s \in\left[t_{\ell}, T\right] .\right.
$$

于是, $\left(\bar{Y}^{k}(\cdot), \bar{Z}^{k}(\cdot)\right)$ 和 $\left(Y^{k}(\cdot), Z^{k}(\cdot)\right)$ 满足同样的 BSDE. 故由唯一性, 必有

$$
\left(\bar{Y}^{k}(s), \bar{Z}^{k}(s)\right)=\left(Y^{k}(s), Z^{k}(s)\right), \quad s \in\left[t_{k}, T\right] .
$$

利用上述同样的方法, 我们可得

$$
\left\{\begin{array}{l}
Y^{k}(s)=\bar{Y}^{k}(s)=\Theta^{k}(s, X(s)), \\
Z^{k}(s)=\bar{Z}^{k}(s)=\sigma(s, X(s)) \Theta^{k}(s, X(s)),
\end{array} s \in\left[t_{k}, T\right],\right.
$$

其中 $\Theta^{k}(\cdot, \cdot)$ 满足下述方程:

$$
\left\{\begin{array}{cl}
\Theta_{s}^{k}(s, x)+\frac{1}{2} \sigma(s, x)^{2} \Theta_{x x}^{k}(s, x)+b(s, x) \Theta_{x}^{k}(s, x) & \\
\quad+g\left(t_{k}, s, x, \Theta^{\ell}(s, x), \sigma(s, x) \Theta_{x}^{k}(s, x)\right)=0, & (s, x) \in\left(t_{\ell}, t_{\ell+1}\right], \quad k \leqslant \ell \leqslant N-1, \\
\Theta^{k}(T, x)=\psi\left(t_{k}, x\right), & x \in \mathbb{R} .
\end{array}\right.
$$

假如令

$$
\Theta^{\Pi}(t, s, x)=\sum_{k=0}^{N-1} \Theta^{k}(s, x) I_{\left(t_{k}, t_{k+1}\right]}(t), \quad(t, s, x) \in \Delta \times \mathbb{R}
$$

则有

$$
\begin{aligned}
Y^{\Pi}(s) & =Y^{0}(s) I_{\left[0, t_{1}\right]}(s)+\sum_{k=0}^{N-1} Y^{k}(s) I_{\left(t_{k}, t_{k+1}\right]}(s) \\
& =\Theta^{0}(s, X(s)) I_{\left[0, t_{1}\right]}(s)+\sum_{k=0}^{N-1} \Theta^{k}(s, X(s)) I_{\left(t_{k}, t_{k+1}\right]}(s) \\
& =\Theta^{\Pi}(s, s, X(s)), \quad s \in[0, T],
\end{aligned}
$$

同时也有

$$
\begin{aligned}
Z^{\Pi}(t, s) & =Z^{0}(s) I_{\left[0, t_{1}\right]}(t)+\sum_{k=0}^{N-1} Z^{k}(s) I_{\left(t_{k}, t_{k+1}\right]}(t) \\
& =\sigma(s, X(s)) \Theta_{x}^{0}(s, X(s)) I_{\left[0, t_{1}\right]}(t)+\sum_{k=0}^{N-1} \sigma(s, X(s)) \Theta_{x}^{k}(s, X(s)) I_{\left(t_{k}, t_{k+1}\right]}(t) \\
& =\sigma(s, X(s)) \Theta_{x}^{\Pi}(t, s, X(s)), \quad(t, s) \in \Delta
\end{aligned}
$$

而对于任何 $t \in[0, T)$, 设 $t \in\left[t_{k}, t_{k+1}\right)$, 我们有

$$
\begin{gathered}
\Theta_{s}^{\Pi}(t, s, x)+\frac{1}{2} \sigma(s, x)^{2} \Theta_{x x}^{\Pi}(t, s, x)+b(s, x) \Theta^{\Pi}(t, s, x) \\
+g\left(\tau^{\Pi}(t), s, x, \Theta^{\Pi}(s, s, x), \sigma(s, x) \Theta_{x}^{\Pi}(t, s, x)\right)=0 .
\end{gathered}
$$


可以期望

$$
\lim _{\|\Pi\| \rightarrow 0} \Theta^{\Pi}(t, s, x)=\Theta(t, s, x)
$$

而 $\Theta(t, s, x)$ 应该满足 (1.6), 并且 BSVIE (1.4) 的适应解 $(Y(\cdot), Z(\cdot, \cdot))$ 应具有表示 $(1.5)$. 事实上, 我们 能够证明如下定理 (这是定理 1.1 的一个较为细致的复述).

定理 3.1 假定 (H1) 和 (H2) 成立, 则 BSVIE (1.4) 存在一个唯一的适应解 $(Y(\cdot), Z(\cdot, \cdot))$, 偏微 分方程 (1.6) 存在唯一的经典解 $\Theta(t, s, x)$. 进一步, 表示式 (1.5) 成立, 其中 $X(\cdot)$ 为 $\operatorname{FSDE}(1.3)$ 的唯 一解.

证明思路 在条件 $(\mathrm{H} 1)$ 和 $(\mathrm{H} 2)$ 下, 我们首先证明 $(1.6)$ 存在唯一的经典解 $\Theta(\cdot, \cdot, \cdot)$. 为此, 我们 任取一个函数 $\theta:[0, T] \times \mathbb{R} \rightarrow \mathbb{R}$, 考虑下述方程:

$$
\begin{cases}\Theta_{s}(t, s, x)+\frac{1}{2} \sigma(s, x)^{2} \Theta_{x x}(t, s, x)+b(s, x) \Theta_{x}(t, s, x) & \\ \quad+g\left(t, s, x, \theta(s, x), \Theta_{x}(t, s, x) \sigma(s, x)\right)=0, & (t, s, x) \in \Delta \times \mathbb{R} \\ \Theta(t, T, x)=\psi(t, x), & (t, x) \in[0, T] \times \mathbb{R} .\end{cases}
$$

这个方程存在唯一的经典解. 于是我们定义了一个映照 $\theta(s, x) \mapsto \Theta(s, s, x)$. 可以证明这个映照具有唯 一的不动点. 从而得到方程 (1.6) 存在唯一的经典解, 并且它是关于系数具有连续依赖性. 从而, 可以 得到 $\Theta^{\Pi}(\cdot, \cdot, \cdot)$ 收玫于 $\Theta(\cdot, \cdot, \cdot)$. 于是, 再用 $\left(Y^{\Pi}(\cdot), Z^{\Pi}(\cdot, \cdot)\right)$ 的表示和定理 2.1 , 我们最后得到 $(1.5)$.

从表示式 (1.5), 我们可见当 $(\mathrm{H} 1)$ 和 $(\mathrm{H} 2)$ 成立时, $(t, s) \mapsto Z(t, s)$ 是连续的. 特别地, $s \mapsto Z(t, s)$ 是连续的. 这样的结果不借助于上述的表示式似乎是无法得到的.

\section{4 推广情形}

本节将简单叙述若干推广情形.

(1) 扩散退化情形: 假定在 $(\mathrm{H} 1)$ 中, $\sigma_{0}=0$, 则我们面临 FSDE 扩散系数退化的情形. 由于我们考 虑的是一维情形, 因此, 黏性解的理论在此可以发挥作用. 具体地, 我们任取 $\varepsilon>0$, 引入下述 FSDE:

$$
\left\{\begin{array}{l}
d X^{\varepsilon}(s)=b\left(s, X^{\varepsilon}(s)\right) d s+\left[\sigma\left(s, X^{\varepsilon}(s)\right)+\varepsilon\right] d W(s), \quad s \in[0, T] \\
X^{\varepsilon}(0)=x
\end{array}\right.
$$

在 $(\mathrm{H} 1)$ 条件假设下, 上述 FSDE 存在唯一解 $X^{\varepsilon}(\cdot)$, 并且

$$
\mathbb{E}\left[\sup _{s \in[0, T]}\left|X^{\varepsilon}(s)-X(s)\right|^{2}\right] \leqslant K \sqrt{\varepsilon}
$$

其中 $X(\cdot)$ 是 $\operatorname{FSDE}(1.3)$ 的解. 然后, 我们引入下述 BSVIE:

$$
Y^{\varepsilon}(t)=\psi\left(t, X^{\varepsilon}(T)\right)+\int_{t}^{T} g\left(t, s, X^{\varepsilon}(s), Y^{\varepsilon}(s), Z^{\varepsilon}(t, s)\right) d s-\int_{t}^{T} Z^{\varepsilon}(t, s) d W(s), \quad s \in[0, T] .
$$


易知,

$$
\begin{aligned}
\mathbb{E}\left(\int_{0}^{T}\left|Y^{\varepsilon}(t)-Y(t)\right|^{2} d t+\int_{0}^{T} \int_{t}^{T}\left|Z^{\varepsilon}(t, s)-Z(t, s)\right|^{2} d s d t\right) \\
\leqslant K \mathbb{E}\left(\left|\psi\left(t, X^{\varepsilon}(T)\right)-\psi(t, X(T))\right|^{2}+\int_{0}^{T} \int_{t}^{T} \mid g\left(t, s, X^{\varepsilon}(s), Y(s), Z(t, s)\right)\right. \\
\left.\quad-\left.g(t, s, X(s), Y(s), Z(t, s))\right|^{2} d s d t\right) \\
\leqslant K \mathbb{E}\left(\left|X^{\varepsilon}(T)-X(T)\right|^{2}+\int_{t}^{T} \int_{t}^{T}\left|X^{\varepsilon}(s)-X(s)\right|^{2} d s d t\right) \leqslant K \varepsilon .
\end{aligned}
$$

于是, 利用定理 3.1 , 我们可得如下表示:

$$
\left\{\begin{array}{l}
Y^{\varepsilon}(s)=\Theta^{\varepsilon}\left(s, s, X^{\varepsilon}(s)\right), \\
Z^{\varepsilon}(t, s)=\left[\sigma\left(s, X^{\varepsilon}(s)\right)+\varepsilon\right] \Theta_{x}^{\varepsilon}\left(t, s, X^{\varepsilon}(s)\right),
\end{array} s \in[0, T],\right.
$$

其中 $(s, x) \mapsto \Theta^{\varepsilon}(t, s, x)$ 满足下述方程:

$$
\left\{\begin{array}{cl}
\Theta_{s}^{\varepsilon}(t, s, x)+\frac{1}{2}[\sigma(s, x)+\varepsilon]^{2} \Theta_{x x}^{\varepsilon}(t, s, x)+b(s, x) \Theta_{x}^{\varepsilon}(t, s, x) & \\
+g\left(t, s, x, \Theta^{\varepsilon}(s, s, x), \Theta_{x}^{\varepsilon}(t, s, x)(\sigma(s, x)+\varepsilon)\right)=0, & (t, s, x) \in \Delta \times \mathbb{R}, \\
\Theta^{\varepsilon}(t, T, x)=\psi(t, x), & (t, x) \in[0, T] \times \mathbb{R} .
\end{array}\right.
$$

最后, 我们令 $\varepsilon \rightarrow 0$. 此时, $\Theta^{\varepsilon}(\cdot, \cdot, \cdot) \rightarrow \Theta(\cdot, \cdot, \cdot)$, 而 $(s, x) \mapsto \Theta(t, s, x)$ 是方程 (1.6) 唯一的黏性解. 值得 注意, 由于 FSDE (1.3) 的扩散项可能退化, 于是 (1.6) 可能没有经典解. 因此, 很自然地, 我们会想到 利用黏性解的理论. 不过, 需要指出的是, 我们的方程 (1.6) 中, 除了 $\Theta(t, s, x) 、 \Theta_{x}(t, s, x)$ 和 $\Theta_{x x}(t, s, x)$ 外, 只出现 $\Theta(s, s, x)$, 而 $\Theta_{x}(s, s, x)$ 和 $\Theta_{x x}(s, s, x)$ 并不出现. 因此, 将 $t$ 看作参数, 整套的黏性解理论 可以照搬. 假如 $\Theta_{x}(s, s, x)$ 或 $\Theta_{x x}(s, s, x)$ 也同时出现, 则我们暂时不知是否可以建立相应的黏性解理 论. 有关黏性解的理论, 限于篇幅, 我们在这里就不展开了, 读者可以参见文献 [17,18].

(2) 高维情形: 对于高维情形, 我们来简单看一下相关结论的表述. 此时, 我们假定 Brown 运动 $W(\cdot)$ 是 $d$ 维的, $X(\cdot)$ 取值于 $\mathbb{R}^{n}, Y(\cdot)$ 取值于 $\mathbb{R}^{m}, Z(\cdot, \cdot)$ 取值于 $\mathbb{R}^{m \times d}$. 相应地, 我们有

$$
\begin{aligned}
& b:[0, T] \times \mathbb{R}^{n} \rightarrow \mathbb{R}^{n}, \quad \sigma:[0, T] \times \mathbb{R}^{n} \rightarrow \mathbb{R}^{n \times d}, \\
& g: \Delta \times \mathbb{R}^{n} \times \mathbb{R}^{m} \times \mathbb{R}^{m \times d} \rightarrow \mathbb{R}^{m}, \quad \psi:[0, T] \times \mathbb{R}^{n} \rightarrow \mathbb{R}^{m} .
\end{aligned}
$$

(H1) 和 (H2) 中的条件也需作相应调整, 例如, 我们需要假定

$$
\sigma(t, x) \sigma(t, x)^{\mathrm{T}} \geqslant \sigma_{0} I, \quad \forall(t, x) \in[0, T] \times \mathbb{R}^{n} .
$$

对于高维情形, 我们尚不知如何处理退化情形. 因此, 上面条件中, 我们需要 $\sigma_{0}>0$. 这样, 必须假定 $d \geqslant n$. 此时, 对应的偏微分方程 (1.6) 将变成如下的偏微分方程组:

$$
\left\{\begin{aligned}
\Theta_{s}(t, s, x)+\frac{1}{2} \sigma(s, x)^{\mathrm{T}} \Theta_{x x}(t, s, x) \sigma(s, x)+\Theta_{x}(t, s, x) b(s, x) & \\
\quad+g\left(t, s, x, \Theta(s, s, x), \Theta_{x}(t, s, x) \sigma(s, x)\right)=0, & (t, s, x) \in \Delta \times \mathbb{R}^{n}, \\
\Theta(t, T, x)=\psi(t, x), & (t, x) \in[0, T] \times \mathbb{R}^{n},
\end{aligned}\right.
$$


其中

$$
\sigma(s, x)^{\mathrm{T}} \Theta_{x x}(t, s, x) \sigma(s, x)=\sum_{k=1}^{d}\left(\begin{array}{c}
\sigma_{k}(s, x)^{\mathrm{T}} \Theta_{x x}^{1}(t, s, x) \sigma_{k}(s, x) \\
\sigma_{k}(s, x)^{\mathrm{T}} \Theta_{x x}^{2}(t, s, x) \sigma_{k}(s, x) \\
\vdots \\
\sigma_{k}(s, x)^{\mathrm{T}} \Theta_{x x}^{m}(t, s, x) \sigma_{k}(s, x)
\end{array}\right),
$$

并且

$$
\sigma(s, x)=\left(\sigma_{1}(s, x), \sigma_{2}(s, x), \ldots, \sigma_{d}(s, x)\right), \quad \Theta(t, s, x)=\left(\begin{array}{c}
\Theta^{1}(t, s, x) \\
\Theta^{2}(t, s, x) \\
\vdots \\
\Theta^{m}(t, s, x)
\end{array}\right) .
$$

此时, 相应的 BSVIE 的适应解 $(Y(\cdot), Z(\cdot, \cdot))$ 的表示应写成如下形式:

$$
\begin{cases}Y(s)=\Theta(s, s, X(s)), & s \in[0, T] \\ Z(t, s)=\Theta_{x}(t, s, X(s)) \sigma(s, X(s)), & (t, s) \in \Delta,\end{cases}
$$

其中 $\Theta(t, s, x)$ 和 $\Theta_{x}(t, s, x)$ 分别取值于 $\mathbb{R}^{m}$ 和 $\mathbb{R}^{m \times n}$.

\section{参考文献}

1 Bismut J M. Théorie Probabiliste du Contrôle des Diffusions. Mem Amer Math Soc, vol. 176. Providence: Amer Math Soc, 1973

2 Pardoux E, Peng S. Adapted solution of a backward stochastic differential equation. Systems Control Lett, 1990, 14: $55-61$

3 El Karoui N, Peng S, Quenez M C. Backward stochastic differential equation in finance. Math Finance, 1997, 7: 1-71

4 Ma J, Protter P, Yong J. Solving forward-backward stochastic differential equations explicitly-A four step scheme. Probab Theory Related Fields, 1994, 98: 339-359

5 Ma J, Yong J. Forward-backward stochastic differential equations and their applications. Lecture Notes in Mathematics, vol. 1702. Berlin: Springer-Verlag, 1999

6 Ma J, Zhang J. Representation theorems for backward stochastic differential equations. Ann Appl Probab, 2002, 12: 1390-1418

7 Zhang J. A numerical scheme for BSDEs. Ann Appl Probab, 2004, 14: 459-488

8 Ma J, Yong J, Zhao Y. Four step scheme for general Markovian forward-backward SDEs. J Syst Sci Complex, 2010, 23: 546-571

9 Peng S. Backward stochastic differential equation, nonlinear expectation and their applications. In: Proceedings of the International Congress of Mathematicians, vol. I. New Delhi: Hindustan Book Agency, 2010, 393-432

10 Lin J. Adapted solution of a backward stochastic nonlinear Volterra integral equation. Stoch Anal Appl, 2002, 20: 165-183

11 Yong J. Backward stochastic Volterra integral equations and some related problems. Stochastic Process Appl, 2006, 116: $779-795$

12 Yong J. Continuous-time dynamic risk measures by backward stochastic Volterra integral equations. Appl Anal, 2007, 86: 1429-1442

13 Yong J. Well-posedness and regularity of backward stochastic Volterra integral equations. Probab Theory Related Fields, 2008, 142: 21-77

14 Wang T, Yong J. Comparison theorems for backward stochastic integral equations. Stochastic Process Appl, 2015, 125: 1756-1798 
15 Shi Y, Wang T, Yong J. Mean-field backward stochastic Volterra integral equations. Discrete Contin Dyn Syst, 2013, 18: 1929-1967

16 Shi Y, Wang T, Yong J. Optimal control problems of forward-backward stochastic Volterra integral equations. Math Control Rel Fields, 2015, 5: 613-649

17 Crandall M G, Ishii H, Lions P L. User's guide to viscosity solutions of second order partial differential equations. Bull Amer Math Soc (NS), 1992, 27: 1-67

18 Yong J, Zhou X Y. Stochastic Control: Hamiltonian Systems and HJB Equations. New York: Springer-Verlag, 1999

\section{Representation of adapted solutions to backward stochastic Volterra integral equations}

YONG JiongMin

Abstract In this paper, we establish a representation for the backward components of the adapted solution of a backward stochastic Volterra integral equation, in terms of the solutions to a partial differential equation and a forward stochastic differential equation.

Keywords backward stochastic Volterra integral equation, adapted solution, representation MSC(2010) 60H20, 60H10, $45 \mathrm{H} 05$

doi: 10.1360/N012016-00034 